\title{
Statistical Riemann and Lebesgue Integrable Sequence of Functions with Korovkin-Type Approximation Theorems
}

\author{
Hari Mohan Srivastava ${ }^{1,2,3,4, * \mathbb{D}}$, Bidu Bhusan Jena ${ }^{5}$ and Susanta Kumar Paikray ${ }^{5}$ \\ 1 Department of Mathematics and Statistics, University of Victoria, Victoria, BC V8W 3R4, Canada \\ 2 Department of Medical Research, China Medical University Hospital, China Medical University, \\ Taichung 40402, Taiwan \\ 3 Department of Mathematics and Informatics, Azerbaijan University, 71 Jeyhun Hajibeyli Street, \\ Baku AZ1007, Azerbaijan \\ 4 Section of Mathematics, International Telematic University Uninettuno, I-00186 Rome, Italy \\ 5 Department of Mathematics, Veer Surendra Sai University of Technology, Burla 768018, India; \\ bidumath.05@gmail.com (B.B.J.); skpaikray_math@vssut.ac.in (S.K.P.) \\ * Correspondence: harimsri@math.uvic.ca
}

Citation: Srivastava, H.M.; Jena, B.B. Paikray, S.K. Statistical Riemann and Lebesgue Integrable Sequence of Functions with Korovkin-Type Approximation Theorems. Axioms 2021, 10, 229. https://doi.org/ 10.3390 /axioms10030229

Academic Editors: Sidney A. Morris and Silvestru Sever Dragomir

Received: 2 August 2021

Accepted: 13 September 2021

Published: 16 September 2021

Publisher's Note: MDPI stays neutral with regard to jurisdictional claims in published maps and institutional affiliations.

Copyright: (c) 2021 by the authors. Licensee MDPI, Basel, Switzerland. This article is an open access article distributed under the terms and conditions of the Creative Commons Attribution (CC BY) license (https:// creativecommons.org/licenses/by/ $4.0 /)$.
Abstract: In this work we introduce and investigate the ideas of statistical Riemann integrability, statistical Riemann summability, statistical Lebesgue integrability and statistical Lebesgue summability via deferred weighted mean. We first establish some fundamental limit theorems connecting these beautiful and potentially useful notions. Furthermore, based upon our proposed techniques, we establish the Korovkin-type approximation theorems with algebraic test functions. Finally, we present two illustrative examples under the consideration of positive linear operators in association with the Bernstein polynomials to exhibit the effectiveness of our findings.

Keywords: riemann integral; lebesgue integral; statistical convergence; deferred weighted mean; banach space; positive linear operators; bernstein polynomial; Korovkin-type approximation theorems

MSC: Primary 40A05; 40G15; Secondary 41A36

\section{Introduction Preliminaries and Motivation}

Let $[a, b] \subset \mathbb{R}$, for every $k \in \mathbb{N}$ there defined a sequence $\left(h_{k}\right)$ of functions such that $h_{k}:[a, b] \rightarrow \mathbb{R}$.

The Riemann sum $\delta\left(h_{k} ; \mathcal{P}\right)$ of the sequence $\left(h_{k}\right)$ of functions associated with a tagged partition $\mathcal{P}$ can be viewed as

$$
\delta\left(h_{k} ; \mathcal{P}\right):=\sum_{i=1}^{k} h_{i}\left(\gamma_{i}\right)\left(r_{i}-r_{i-1}\right)
$$

We now recall the notion of Riemann integrability of the sequence of functions on a closed and bounded interval $[a, b]$.

A sequence $\left(h_{k}\right)_{k \in \mathbb{N}}$ of functions is integrable to $h$ (a function) in the Riemann sense over $[a, b]$ if, for each $\epsilon>0, \exists \sigma_{\epsilon}>0$ such that

$$
\left|\delta\left(h_{k} ; \mathcal{P}\right)-h\right|<\epsilon
$$

where $\mathcal{P}$ is any tagged partition of $[a, b]$, and $\|\mathcal{P}\|<\sigma_{\epsilon}$.

We write

$$
h_{k} \in \mathcal{R}[a, b] .
$$

Now, we define the Lebesgue integral of a sequence of measurable functions. 
Let $(X, \mathcal{F}, \lambda)$ be a finite measurable space, and let $\left(h_{k}\right)$ be the sequence of measurable functions with

$$
h_{k}=\sum_{i=1}^{k} b_{i} \chi_{B_{i}}
$$

where $B_{i}=\left[\alpha: h_{k}(\alpha)=b_{i}\right]$ and $b_{i}$ 's are distinct values of $\left(h_{k}\right)$. Then the Lebesgue integral of $\left(h_{k}\right)$ with respect to measure $\lambda$ is given by

$$
\int_{X} h_{k} d \lambda=\sum_{i=1}^{k} b_{i} \lambda\left(B_{i}\right)
$$

The sequence $\left(h_{k}\right)$ of measurable functions is Lebesgue integrable to a measurable function $h$ if, for each $\epsilon>0$

$$
\left|\int_{X} h_{k} d \lambda-h\right|<\epsilon .
$$

We write

$$
h_{k} \in \mathcal{L}(X, \lambda) \text {. }
$$

In sequence spaces, the theory of usual convergence is one of the most essential parts, and gradually it has been achieved a very high level of development. Subsequently, two eminent mathematicians Fast [1] and Steinhaus [2] independently introduced a new concept called statistical convergence in sequence space theory. Really, this nice concept is very useful for advanced study in pure and applied Mathematics. Moreover, it is more powerful than the usual convergence and has been an active area of research in the current days. Moreover, such notion is closely related with the study of Measure theory, Probability theory, Fibonacci sequence, and Real analysis, etc. For some recent research works in this direction, see [3-6].

Suppose $\mathfrak{E} \subseteq \mathbb{N}$, and let $\mathfrak{E}_{k}=\{\eta: \eta \leqq k$ and $\eta \in \mathfrak{E}\}$. Then the natural density $d(\mathfrak{E})$ of $\mathfrak{E}$ is defined by

$$
d(\mathfrak{E})=\lim _{k \rightarrow \infty} \frac{\left|\mathfrak{E}_{k}\right|}{k}=\tau,
$$

where $\left|\mathfrak{E}_{k}\right|$ denotes the cardinality of $\mathfrak{E}_{k}$, and $\tau \in \mathbb{R}$ is finite.

A sequence $\left(u_{n}\right)$ is said to be statistically convergent to $\alpha$ if, for each $\epsilon>0$,

$$
\mathfrak{E}_{\epsilon}=\left\{\eta: \eta \in \mathbb{N} \quad \text { and }\left|u_{\eta}-\alpha\right| \geqq \epsilon\right\}
$$

has natural density (see [1,2]) zero. Thus, for every $\epsilon>0$, we have

$$
d\left(\mathfrak{E}_{\epsilon}\right)=\lim _{k \rightarrow \infty} \frac{\left|\mathfrak{E}_{\epsilon}\right|}{k}=0 .
$$

We write

$$
\text { stat } \lim _{k \rightarrow \infty} u_{k}=\alpha .
$$

In the year 2002, Móricz [7] studied and introduced the notion of statistical Cesàro summability and after that Mohiuddine et al. [8] proved some approximation of the Korovkin-type theorems via the concept of statistical Cesàro summability. Subsequently, Karakaya and Chishti [9] first introduced and studied the idea of weighted statistical convergence, and later this definition was modified by Mursaleen et al. [10]. Recently, Srivastava et al. [11] introduced and studied the concepts of deferred weighted summability mean and proved the Korovkin-type theorems and in the same year Srivastava et al. [12] also proved the Korovkin-type theorems via Nörlund summability mean based on equistatistical convergence. Subsequently, Dutta et al. [13] demonstrated some Korovkin-type approximation theorems via the usual deferred Cesàro summablity mean. Moreover, such concepts have been generalised in many aspects. In view of this, the interested readers may see, [14-19]. 
We now present the notions of statistically Riemann integrable and statistically Lebesgue integrable sequence of functions.

Definition 1. A sequence $\left(h_{k}\right)_{k \in \mathbb{N}}$ of functions is said to be statistically Riemann integrable to $h$ (a function) on $[a, b]$ if, for each $\epsilon>0$ and every $x \in[a, b], \exists \sigma_{\epsilon}>0$, and for any tagged partition $\mathcal{P}$ of $[a, b]\left(\|\mathcal{P}\|<\sigma_{\epsilon}\right)$, the set

$$
\mathfrak{E}_{\epsilon}=\left\{\eta: \eta \in \mathbb{N} \quad \text { and } \quad\left|\delta\left(h_{\eta} ; \mathcal{P}\right)-h\right| \geqq \epsilon\right\}
$$

has zero natural density. That is, for every $\epsilon>0$

$$
d\left(\mathfrak{E}_{\epsilon}\right)=\lim _{k \rightarrow \infty} \frac{\left|\mathfrak{E}_{\epsilon}\right|}{k}=0 .
$$

We write

$$
\text { stat }_{\text {Rie }} \lim _{k \rightarrow \infty} \delta\left(h_{k} ; \mathcal{P}\right)=h .
$$

Definition 2. A sequence $\left(h_{k}\right)_{k \in \mathbb{N}}$ of measurable functions is said to be statistically Lebesgue integrable to a measurable function $h$ on $X$ if, for each $\epsilon>0$

$$
\mathfrak{E}_{\epsilon}=\left\{\eta: \eta \in \mathbb{N} \text { and }\left|\int_{X} h_{k} d \lambda-h\right| \geqq \epsilon\right\}
$$

has zero natural density. That is, for every $\epsilon>0$

$$
d\left(\mathfrak{E}_{\epsilon}\right)=\lim _{k \rightarrow \infty} \frac{\left|\mathfrak{E}_{\epsilon}\right|}{k}=0 .
$$

We write

$$
\text { stat }_{\mathrm{Leb}} \lim _{k \rightarrow \infty} \int_{X} h_{k} d \lambda=h .
$$

Now, we establish a theorem (below) connecting the above two potential and useful concepts.

Theorem 1. If a sequence $\left(h_{k}\right)$ of measurable functions is statistically Riemann integrable to a function $h$ over $[a, b]$, then $\left(h_{k}\right)$ is statistically Lebesgue integrable to the same function $h$ on $X$.

Proof. Suppose a sequence $\left(h_{k}\right)$ of functions is statistically Riemann integrable to a function $h$. Then, for all $\epsilon>0$ and for any tagged partition $\mathcal{P}$ of $[a, b]$ such that $\|\mathcal{P}\|<\sigma_{\epsilon}$, we have

$$
\text { stat }_{\text {Rie }} \lim _{k \rightarrow \infty} \delta\left(h_{k} ; \mathcal{P}\right)=h .
$$

Moreover, $\left(h_{k}\right)$ being a sequence of measurable functions, for every $\epsilon>0$

$$
\int_{X} h_{k} d \lambda \leq \lim _{k \rightarrow \infty} \delta\left(h_{k} ; \mathcal{P}\right) .
$$

Therefore, for each $\epsilon>0$

$$
\begin{aligned}
\lim _{k \rightarrow \infty} \frac{1}{k} \mid\left\{\eta: \eta \in \mathbb{N} \text { and }\left|\int_{X} h_{k} d \lambda-h\right| \geqq \epsilon\right\} \mid \\
\quad \leqq \lim _{k \rightarrow \infty} \frac{1}{k} \mid\left\{\eta: \eta \in \mathbb{N} \text { and }\left|\delta\left(h_{k} ; \mathcal{P}\right)-h\right| \geqq \epsilon\right\} \mid .
\end{aligned}
$$

Consequently, by Definition 2

$$
\text { stat }_{\text {Leb }} \lim _{k \rightarrow \infty} \int_{X} h_{k} d \lambda=h .
$$


We setup here an example demonstrating the non-validity of converse statement of Theorem 1.

Example 1. Let $h_{k}:[0,1] \rightarrow \mathbb{R}$ be the functions defined by $h_{k}(x)=0$ if, $x \in \mathbb{Q} \cap[0,1]$ and $h_{k}(x)=1$ if, $x \in(\mathbb{R} \backslash \mathbb{Q}) \cap[0,1]$.

It is easy to see that the sequence $\left(h_{k}\right)$ of functions is not statistically Riemann integrable, but it is statistically Lebesgue integrable to 1 over $[0,1]$.

Motivated essentially by the aforementioned studies and investigations, we introduce and investigate the notions of statistical versions of Riemann integrability and Riemann summability as well as statistical versions of Lebesgue integrability and Lebesgue summability via deferred weighted mean. We first establish some fundamental limit theorems connecting these beautiful and potentially useful notions. Moreover, based upon our proposed techniques we establish the Korovkin-type approximation theorems with algebraic test functions. Finally, we consider two illustrative examples involving suitable positive linear operators associated with the Bernstein polynomials to justify the effectiveness of our findings.

\section{Riemann Integrability via Deferred Weighted Mean}

Let $\left(\phi_{k}\right)$ and $\left(\varphi_{k}\right) \in \mathbb{Z}^{0+}$ be such that $\phi_{k}<\varphi_{k}$ with $\lim _{k \rightarrow \infty} \varphi_{k}=+\infty$, and let $\left(p_{i}\right)$ be a sequence of real numbers (non-negative) such that

$$
P_{k}=\sum_{i=\phi_{k}+1}^{\varphi_{k}} p_{i}
$$

Then, we approach the Riemann sum of the functions $\delta\left(h_{k} ; \mathcal{P}\right)$ corresponding to a tagged partition $\mathcal{P}$ via the deferred weighted summability mean of the form

$$
\mathcal{W}\left(\delta\left(h_{k} ; \mathcal{P}\right)\right)=\frac{1}{P_{k}} \sum_{\varrho=\phi_{k}+1}^{\varphi_{k}} p_{\varrho} \delta\left(h_{\varrho} ; \mathcal{P}\right)
$$

We now present the definitions of statistical Riemann integrability and statistical Riemann summability via deferred weighted summability mean.

Definition 3. Let $\left(\phi_{k}\right)$ and $\left(\varphi_{k}\right) \in \mathbb{Z}^{0+}$, and let $\left(p_{k}\right)$ be a sequence of real numbers (non-negative). A sequence of functions $\left(h_{k}\right)_{k \in \mathbb{N}}$ is said to be deferred weighted statistically Riemann integrable to $h$ over $[a, b]$ if, for every $\epsilon>0, \exists \sigma_{\epsilon}>0$, and for $\mathcal{P}$ be any tagged partition of $[a, b]$ such that $\|\mathcal{P}\|<\sigma_{\epsilon}$, the set

$$
\left\{\eta: \eta \leqq P_{k} \quad \text { and } \quad p_{\eta}\left|\delta\left(h_{\eta} ; \mathcal{P}\right)-h\right| \geqq \epsilon\right\}
$$

has zero natural density. This implies that, for each $\epsilon>0$,

$$
\lim _{k \rightarrow \infty} \frac{\mid\left\{\eta: \eta \leqq P_{k} \quad \text { and } \quad p_{\eta}\left|\delta\left(h_{\eta} ; \mathcal{P}\right)-k\right| \geqq \epsilon\right\} \mid}{P_{k}}=0 .
$$

We write

$$
D W R_{\text {stat }} \lim _{k \rightarrow \infty} \delta\left(h_{k} ; \mathcal{P}\right)=h
$$

Definition 4. Let $\left(\phi_{k}\right)$ and $\left(\varphi_{k}\right) \in \mathbb{Z}^{0+}$, and let $\left(p_{k}\right)$ be a sequence of non-negative real numbers. A sequence of functions $\left(h_{k}\right)_{k \in \mathbb{N}}$ is said to be statistically deferred weighted Riemann summable to $h$ over $[a, b]$ if, for every $\epsilon>0, \exists \sigma_{\epsilon}>0$, and for $\mathcal{P}$ be any tagged partition of $[a, b]$ such that $\|\mathcal{P}\|<\sigma_{\epsilon}$, the set

$$
\left\{\eta: \eta \leqq k \quad \text { and } \quad\left|\mathcal{W}\left(\delta\left(h_{\eta} ; \mathcal{P}\right)\right)-h\right| \geqq \epsilon\right\}
$$


has zero natural density. This implies that, for all $\epsilon>0$,

$$
\lim _{k \rightarrow \infty} \frac{\mid\left\{\eta: \eta \leqq k \quad \text { and }\left|\mathcal{W}\left(\delta\left(h_{\eta} ; \mathcal{P}\right)\right)-h\right| \geqq \epsilon\right\} \mid}{k}=0 .
$$

We write

$$
\text { stat }_{\mathrm{DWR}} \lim _{k \rightarrow \infty} \delta\left(h_{k} ; \mathcal{P}\right)=h
$$

Now, we establish an inclusion theorem connecting the above two new potentially useful concepts.

Theorem 2. Let $\left(\phi_{k}\right)$ and $\left(\varphi_{k}\right) \in \mathbb{Z}^{0+}$, and let $\left(p_{k}\right)$ be a sequence of non-negative real numbers. If a sequence $\left(h_{k}\right)_{k \in \mathbb{N}}$ of functions is deferred weighted statistically Riemann integrable to $h$ over $[a, b]$, then it is statistically deferred weighted Riemann summable to $h$ on $[a, b]$, but the converse is not true.

Proof. Since $\left(h_{k}\right)_{k \in \mathbb{N}}$ is deferred weighted statistically Riemann integrable to a function $h$ over $[a, b]$, by Definition 3, we have

$$
\lim _{k \rightarrow \infty} \frac{\mid\left\{\eta: \eta \leqq P_{k} \quad \text { and } \quad p_{\eta}\left|\delta\left(h_{\eta} ; \mathcal{P}\right)-h\right| \geqq \epsilon\right\} \mid}{P_{k}}=0 .
$$

Now under the assumption of the following two sets:

$$
\mathcal{O}_{\epsilon}=\left\{\eta: \eta \leqq P_{k} \quad \text { and } \quad p_{\eta}\left|\delta\left(h_{\eta} ; \mathcal{P}\right)-h\right| \geqq \epsilon\right\}
$$

and

$$
\mathcal{O}_{\epsilon}^{c}=\left\{\eta: \eta \leqq P_{k} \quad \text { and } \quad p_{\eta}\left|\delta\left(h_{\eta} ; \mathcal{P}\right)-h\right|<\epsilon\right\},
$$

we have

$$
\begin{aligned}
\left|\mathcal{W}\left(\delta\left(h_{k} ; \mathcal{P}\right)\right)-h\right| & =\left|\frac{1}{P_{k}} \sum_{\varrho=\phi_{k}+1}^{\varphi_{k}} p_{\varrho} \delta\left(h_{\varrho} ; \mathcal{P}\right)-h\right| \\
& \leqq\left|\frac{1}{P_{k}} \sum_{\varrho=\phi_{k}+1}^{\varphi_{k}} p_{\varrho}\left[\delta\left(h_{\varrho} ; \mathcal{P}\right)-h\right]\right|+\left|\frac{1}{P_{k}} \sum_{\varrho=\phi_{k}+1}^{\varphi_{k}} p_{\varrho} h-h\right| \\
& \leqq \frac{1}{P_{k}} \sum_{\substack{\varrho=\phi_{k}+1 \\
\left(\eta \in \mathcal{O}_{\epsilon}\right)}}^{\varphi_{k}} p_{\varrho}\left|\delta\left(h_{\varrho} ; \mathcal{P}\right)-h\right|+\frac{1}{P_{k}} \sum_{\substack{\varrho=\phi_{k}+1 \\
\left(\eta \in \mathcal{O}_{\epsilon}^{c}\right)}}^{\varphi_{k}} p_{\varrho}\left|\delta\left(h_{\varrho} ; \mathcal{P}\right)-h\right| \\
& +|h|\left|\frac{1}{P_{k}} \sum_{\varrho=\phi_{k}+1}^{\varphi_{k}} p_{\varrho}-1\right| \\
& \leqq \frac{1}{P_{k}}\left|\mathcal{O}_{\epsilon}\right|+\frac{1}{P_{k}}\left|\mathcal{O}_{\epsilon}^{c}\right| .
\end{aligned}
$$

This implies that

$$
\left|\mathcal{W}\left(\delta\left(h_{k} ; \mathcal{P}\right)\right)-h\right|<\epsilon .
$$

Thus, the functions $\left(h_{k}\right)$ is statistically deferred weighted Riemann summable to $h$ on $[a, b]$.

Next, in view of the converse statement (non-validity), we consider the following illustrative example.

Example 2. Let $\phi_{k}=2 k, \varphi_{k}=4 k$ and $p_{k}=1$ and let $h_{k}:[0,1] \rightarrow \mathbb{R}$ be the functions of the form $h_{k}(x)=0$, if $x \in \mathbb{Q} \cap[0,1]$ with $k$ is even and $h_{k}(x)=1$, if $x \in \mathbb{R} \backslash \mathbb{Q} \cap[0,1]$ with $k$ is odd. 
The given sequence of functions $\left(h_{k}\right)$ trivially spcifies that, it is neither Riemann integrable nor deferred weighted Riemann integrable in statistical sense. However, according to Equation (1),

$$
\begin{aligned}
\mathcal{W}\left(\delta\left(h_{k} ; \mathcal{P}\right)\right) & =\frac{1}{\varphi_{k}-\phi_{k}} \sum_{\varrho=\phi_{k}+1}^{\varphi_{k}} \delta\left(h_{\varrho} ; \mathcal{P}\right) \\
& =\frac{1}{2 k} \sum_{m=2 k+1}^{4 k} \delta\left(h_{\varrho} ; \mathcal{P}\right)=\frac{1}{2}
\end{aligned}
$$

Thus, the functions $\left(h_{k}\right)$ has deferred weighted Riemann sum $\frac{1}{2}$ corresponding to a tagged partition $\mathcal{P}$. Therefore, the functions $\left(h_{k}\right)$ is statistically deferred weighted Riemann summable to $\frac{1}{2}$ on $[0,1]$ but it is not deferred weighted statistically Riemann integrable.

\section{Lebesgue Integrability via Deferred Weighted Mean}

Let $\left(\phi_{k}\right)$ and $\left(\varphi_{k}\right) \in \mathbb{Z}^{0+}$ be such that $\phi_{k}<\varphi_{k}$ with $\lim _{k \rightarrow \infty} \varphi_{k}=+\infty$, and let $\left(p_{i}\right)$ be a sequence of non-negative real numbers for which

$$
P_{k}=\sum_{i=\phi_{k}+1}^{\varphi_{k}} p_{i}
$$

Then, we define the Lebesgue sum via deferred weighted summability mean for the sequence of measurable functions $\left(h_{k}\right)$ as

$$
\mathcal{W}(\mathcal{L}(X, \lambda)))=\frac{1}{P_{k}} \sum_{\varrho=\phi_{k}+1}^{\varphi_{k}} p_{\varrho}\left(b_{\varrho} \lambda\left(B_{\varrho}\right)\right) .
$$

We now present below the definitions of statistical Lebesgue integrability and statistical Lebesgue summability of a sequence of measurable functions via deferred weighted mean.

Definition 5. Let $\left(\phi_{k}\right)$ and $\left(\varphi_{k}\right) \in \mathbb{Z}^{0+}$, and let $\left(p_{k}\right)$ be a sequence of non-negative real numbers. A sequence $\left(h_{k}\right)_{k \in \mathbb{N}}$ of measurable functions is said to be deferred weighted statistically Lebesgue integrable to a measurable function $h$ on $X$ if, for every $\epsilon>0$, the set

$$
\left\{\eta: \eta \leqq P_{k} \quad \text { and } \quad p_{\eta}\left|b_{\eta} \lambda\left(B_{\eta}\right)-h\right| \geqq \epsilon\right\}
$$

has zero natural density. This implies that, for each $\epsilon>0$,

$$
\lim _{k \rightarrow \infty} \frac{\mid\left\{\eta: \eta \leqq P_{k} \quad \text { and } \quad p_{\eta}\left|b_{\eta} \lambda\left(B_{\eta}\right)-h\right| \geqq \epsilon\right\} \mid}{P_{k}}=0 .
$$

We write

$$
D W L_{\text {stat }} \lim _{k \rightarrow \infty} h_{k}=h
$$

Definition 6. Let $\left(\phi_{k}\right)$ and $\left(\varphi_{k}\right) \in \mathbb{Z}^{0+}$, and let $\left(p_{k}\right)$ be a sequence of non-negative real numbers. A sequence $\left(h_{k}\right)_{k \in \mathbb{N}}$ of measurable functions is statistically deferred weighted Lebesgue summable to a measurable function $h$ on $X$ if, for all $\epsilon>0$

$$
\{\eta: \eta \leqq k \quad \text { and } \quad|\mathcal{W}(\mathcal{L}(X, \lambda))-h| \geqq \epsilon\}
$$

has zero natural density. This implies that, for all $\epsilon>0$,

$$
\lim _{k \rightarrow \infty} \frac{\mid\{\eta: \eta \leqq k \text { and }|\mathcal{W}(\mathcal{L}(X, \lambda))-h| \geqq \epsilon\} \mid}{k}=0 .
$$


We write

$$
\text { stat }_{\mathrm{DWL}} \lim _{k \rightarrow \infty} h_{k}=h
$$

We present below a theorem connecting these two potentially useful concepts.

Theorem 3. Let $\left(\phi_{k}\right)$ and $\left(\varphi_{k}\right) \in \mathbb{Z}^{0+}$, and let $\left(p_{k}\right)$ be a sequence of non-negative real numbers. If a sequence $\left(h_{k}\right)_{k \in \mathbb{N}}$ of measurable functions is deferred weighted statistically Lebesgue integrable to a measurable function $h$ on $X$, then it is statistically deferred weighted Lebesgue summable to the same measurable function $h$ on $X$, but the converse is not true.

Proof. Since $\left(h_{k}\right)_{k \in \mathbb{N}}$ is deferred weighted statistically Lebesgue integrable to a measurable function $h$ on $X$, by Definition 5 , we obtain

$$
\lim _{k \rightarrow \infty} \frac{\mid\left\{\eta: \eta \leqq P_{k} \quad \text { and } \quad p_{\eta}\left|b_{\eta} \lambda\left(B_{\eta}\right)-h\right| \geqq \epsilon\right\} \mid}{P_{k}}=0 .
$$

Now under the assumption of the following two sets:

$$
\mathcal{D}_{\epsilon}=\left\{\eta: \eta \leqq P_{k} \quad \text { and } \quad p_{\eta}\left|b_{\eta} \lambda\left(B_{\eta}\right)-h\right| \geqq \epsilon\right\}
$$

and

$$
\mathcal{D}_{\epsilon}^{c}=\left\{\eta: \eta \leqq P_{k} \quad \text { and } \quad p_{\eta}\left|b_{\eta} \lambda\left(B_{\eta}\right)-h\right|<\epsilon\right\},
$$

we have

$$
\begin{aligned}
&|\mathcal{W}(\mathcal{L}(X, \lambda))-h|=\left|\frac{1}{P_{k}} \sum_{\varrho=\phi_{k}+1}^{\varphi_{k}} p_{\varrho} b_{\varrho} \lambda\left(B_{\varrho}\right)-h\right| \\
& \leqq \\
&\left|\frac{1}{P_{k}} \sum_{\varrho=\phi_{k}+1}^{\varphi_{k}} p_{\varrho}\left[b_{\varrho} \lambda\left(B_{\varrho}\right)-h\right]\right|+\left|\frac{1}{P_{k}} \sum_{\varrho=\phi_{k}+1}^{\varphi_{k}} p_{\varrho} h-h\right| \\
& \leqq \frac{1}{P_{k}} \sum_{\substack{\varrho=\phi_{k}+1 \\
\left(\eta \in \mathcal{D}_{\epsilon}\right)}}^{\varphi_{k}} p_{\varrho}\left|b_{\varrho} \lambda\left(B_{\varrho}\right)-h\right|+\frac{1}{P_{k}} \sum_{\substack{\varrho=\phi_{k}+1 \\
\left(\eta \in \mathcal{D}_{\epsilon}^{c}\right)}}^{\varphi_{k}} p_{\varrho}\left|b_{\varrho} \lambda\left(B_{\varrho}\right)-h\right| \\
&+|h|\left|\frac{1}{P_{k}} \sum_{\varrho=\phi_{k}+1}^{\varphi_{k}} p_{\varrho}-1\right| \\
& \leqq \frac{1}{P_{k}}\left|\mathcal{D}_{\epsilon}\right|+\frac{1}{P_{k}}\left|\mathcal{D}_{\epsilon}^{c}\right|<\epsilon .
\end{aligned}
$$

This implies that

$$
|\mathcal{W}(\mathcal{L}(X, \lambda))-h|<\epsilon .
$$

Hence, the sequence $\left(h_{k}\right)$ of measurable functions is statistically deferred weighted Lebesgue summable to the measurable function $h$ on $X$.

Next in view of the non-validity of the converse statement, the following example illustrates that, a statistically deferred weighted Lebesgue summable sequence of measurable functions is not deferred weighted statistically Lebesgue integrable.

Example 3. Let $\phi_{k}=2 k, \varphi_{k}=4 k$ and $p_{k}=1$ and let $h_{k}:[0,1] \rightarrow \mathbb{R}$ be the functions given by $h_{k}(x)=1$ if, $x \in[0,1]$ with $k$ is even and $h_{k}(x)=0$ if, $x=0$ with $k$ is odd.

The given sequence $\left(h_{k}\right)$ of measurable functions clearly specifies that, it is neither Lebesgue integrable nor deferred weighted statistically Lebesgue integrable. However, according to our proposed mean (2), it is easy to see

$$
\mathcal{W}(\mathcal{L}(X, \lambda))=\frac{1}{\varphi_{k}-\phi_{k}} \sum_{\varrho=\phi_{k}+1}^{\varphi_{k}} b_{\varrho} \lambda\left(B_{\varrho}\right)
$$




$$
=\frac{1}{2 k} \sum_{m=2 k+1}^{4 k} b_{\varrho} \lambda\left(B_{\varrho}\right)=\frac{1}{2} .
$$

Thus, the sequence $\left(h_{k}\right)$ of measurable functions has deferred weighted Lebesgue sum $\frac{1}{2}$. Therefore, the sequence $\left(h_{k}\right)$ of measurable functions is statistically deferred weighted Lebesgue summable to $\frac{1}{2}$ over $[0,1]$ but it is not deferred weighted statistically Lebesgue integrable.

\section{Korovkin-Type Approximation Theorems}

Recently, several researchers have worked to extend (or generalize) the approximation aspects of Korovkin's approximation theorems in various mathematical fields such as (for example) Soft computing, Machine learning, Probability theory, Measurable theory, and so on. This concept is extremely valuable in Real Analysis, Functional Analysis, Harmonic Analysis, and other related fields. Here, we choose to refer to recent works $[11,13,20]$ for interested readers .

Let $C[0,1]$ be the space of all real-valued continuous functions, and evidently it is a complete normed linear space (Banach space) under the sup norm. Then for $h \in C[0,1]$, the sup norm of $h$ is defined as

$$
\|h\|_{\infty}=\sup \{|h(\rho)|: \leqq \rho \leqq 1\} .
$$

Let $\mathfrak{G}_{j}: C[0,1] \rightarrow C[0,1]$ such that

$$
\mathfrak{G}_{j}(h ; \rho) \geqq 0 \quad \text { as } \quad h \geqq 0 .
$$

That is to say, $\mathfrak{G}_{j}$ is a sequence of positive linear operators over $[0,1]$.

Now in view of our proposed mean (1), we use the notion of statistical Riemann integrability $\left(D_{W} R_{\text {stat }}\right)$ and statistical Riemann summability (stat DWR $\left._{\text {R }}\right)$ for sequence of functions to establish and prove the following Korovkin-type approximation theorems.

Theorem 4. Let

$$
\mathfrak{G}_{j}: C[0,1] \rightarrow C[0,1]
$$

be a sequence of positive linear operators. Then, for all $h \in C[0,1]$,

$$
\mathrm{DWR}_{\text {stat }} \lim _{j \rightarrow \infty}\left\|\mathfrak{G}_{j}(h ; \rho)-h(\rho)\right\|_{\infty}=0
$$

if and only if

$$
\begin{aligned}
& \operatorname{DWR}_{\text {stat }} \lim _{j \rightarrow \infty}\left\|\mathfrak{G}_{j}(1 ; \rho)-1\right\|_{\infty}=0, \\
& \operatorname{DWR}_{\text {stat }} \lim _{j \rightarrow \infty}\left\|\mathfrak{G}_{j}(\rho ; \rho)-\rho\right\|_{\infty}=0
\end{aligned}
$$

and

$$
\mathrm{DWR}_{\text {stat }} \lim _{j \rightarrow \infty}\left\|\mathfrak{G}_{j}\left(\rho^{2} ; \rho\right)-\rho^{2}\right\|_{\infty}=0 .
$$

Proof. Since each of the following functions:

$$
h_{0}(\rho)=1, \quad h_{1}(\rho)=2 \rho \quad \text { and } \quad h_{2}(\rho)=3 \rho^{2}
$$

belongs to $C[0,1]$ and is continuous, the implication given by (3) obviously implies (4) to $(6)$. 
Now for the completion of the proof of Theorem 4 , we assume that, the conditions (4) to (6) hold true. If $h \in C[0,1]$, then there exists a constant $\mathcal{K}>0$ such that

$$
|h(\rho)| \leqq \mathcal{K} \quad(\forall \rho \in[0,1]) .
$$

We thus find that

$$
|h(r)-h(\rho)| \leqq 2 \mathcal{K} \quad(r, \rho \in[0,1]) .
$$

Clearly, for given $\epsilon>0$, there exists $\delta>0$ such that

$$
|f(r)-f(\rho)|<\epsilon
$$

whenever

$$
|r-\rho|<\delta, \quad \text { for all } \quad r, \rho \in[0,1] .
$$

Let us choose

$$
\mu_{1}=\mu_{1}(r, \rho)=(2 r-2 \rho)^{2} .
$$

If

$$
|r-\rho| \geqq \delta,
$$

then, we obtain

$$
|h(r)-h(\rho)|<\frac{2 \mathcal{K}}{\theta^{2}} \mu_{1}(r, \rho) .
$$

From Equations (8) and (9), we get

$$
|h(r)-h(\rho)|<\epsilon+\frac{2 \mathcal{K}}{\theta^{2}} \mu_{1}(r, \rho),
$$

which implies that

$$
-\epsilon-\frac{2 \mathcal{K}}{\theta^{2}} \mu_{1}(r, \rho) \leqq h(r)-h(\rho) \leqq \epsilon+\frac{2 \mathcal{K}}{\theta^{2}} \mu_{1}(r, \rho) .
$$

Now, since $\mathfrak{G}_{m}(1 ; \rho)$ is monotone and linear, by applying the operator $\mathfrak{G}_{m}(1 ; \rho)$ to this inequality, we have

$$
\begin{aligned}
\mathfrak{G}_{m}(1 ; \rho)\left(-\epsilon-\frac{2 \mathcal{K}}{\theta^{2}} \mu_{1}(r, \rho)\right) & \leqq \mathfrak{G}_{m}(1 ; \rho)(h(r)-h(\rho)) \\
& \leqq \mathfrak{G}_{m}(1 ; \rho)\left(\epsilon+\frac{2 \mathcal{K}}{\theta^{2}} \mu_{1}(r, \rho)\right) .
\end{aligned}
$$

We note that $\rho$ is fixed and so $h(\rho)$ is a constant number. Therefore, we have

$$
\begin{aligned}
-\epsilon \mathfrak{G}_{m}(1 ; \rho)-\frac{2 \mathcal{K}}{\theta^{2}} \mathfrak{G}_{m}\left(\mu_{1} ; \rho\right) & \leqq \mathfrak{G}_{m}(h ; \rho)-h(\rho) \mathfrak{G}_{m}(1 ; \rho) \\
& \leqq \epsilon \mathfrak{G}_{m}(1 ; \rho)+\frac{2 \mathcal{K}}{\theta^{2}} \mathfrak{G}_{m}\left(\mu_{1} ; \rho\right) .
\end{aligned}
$$

Moreover, we know that

$$
\mathfrak{G}_{m}(h ; \rho)-h(\rho)=\left[\mathfrak{G}_{m}(h ; \rho)-h(\rho) \mathfrak{G}_{m}(1 ; \rho)\right]+h(\rho)\left[\mathfrak{G}_{m}(1 ; \rho)-1\right] .
$$

Using (11) and (12), we have

$$
\mathfrak{G}_{m}(h ; \rho)-h(\rho)<\epsilon \mathfrak{G}_{m}(1 ; \rho)+\frac{2 \mathcal{K}}{\theta^{2}} \mathfrak{G}_{m}\left(\mu_{1} ; \rho\right)+h(\rho)\left[\mathfrak{G}_{m}(1 ; \rho)-1\right] .
$$


We now estimate $\mathfrak{G}_{m}\left(\mu_{1} ; \rho\right)$ as follows:

$$
\begin{aligned}
\mathfrak{G}_{m}\left(\mu_{1} ; \rho\right)= & \mathfrak{G}_{m}\left((2 r-2 \rho)^{2} ; \rho\right)=\mathfrak{G}_{m}\left(2 r^{2}-8 \rho r+4 \rho^{2} ; \rho\right) \\
= & \mathfrak{G}_{m}\left(4 r^{2} ; \rho\right)-8 t \mathfrak{G}_{m}(r ; \rho)+4 \rho^{2} \mathfrak{G}_{m}(1 ; \rho) \\
= & 4\left[\mathfrak{G}_{m}\left(r^{2} ; \rho\right)-\rho^{2}\right]-8 t\left[\mathfrak{G}_{m}(r ; \rho)-\rho\right] \\
& +4 \rho^{2}\left[\mathfrak{G}_{m}(1 ; \rho)-1\right] .
\end{aligned}
$$

Using (13), we obtain

$$
\begin{aligned}
\mathfrak{G}_{m}(h ; \rho)-h(\rho)<\epsilon & \mathfrak{G}_{m}(1 ; \rho)+\frac{2 \mathcal{K}}{\theta^{2}}\left\{4\left[\mathfrak{G}_{m}\left(r^{2} ; \rho\right)-\rho^{2}\right]\right. \\
& \left.-8 \rho\left[\mathfrak{G}_{m}(r ; \rho)-\rho\right]+4 \rho^{2}\left[\mathfrak{G}_{m}(1 ; \rho)-1\right]\right\} \\
& +h(\rho)\left[\mathfrak{G}_{m}(1 ; \rho)-1\right] . \\
=\epsilon & {\left[\mathfrak{G}_{m}(1 ; \rho)-1\right]+\epsilon+\frac{2 \mathcal{K}}{\theta^{2}}\left\{4\left[\mathfrak{G}_{m}\left(r^{2} ; \rho\right)-\rho^{2}\right]\right.} \\
& \left.-8 \rho\left[\mathfrak{G}_{m}(r ; \rho)-\rho\right]+4 \rho^{2}\left[\mathfrak{G}_{m}(1 ; \rho)-1\right]\right\} \\
& +h(\rho)\left[\mathfrak{G}_{m}(1 ; \rho)-1\right] .
\end{aligned}
$$

Since $\epsilon>0$ is arbitrary, we can write

$$
\begin{aligned}
\left|\mathfrak{G}_{m}(h ; \rho)-h(\rho)\right| \leqq & \epsilon+\left(\epsilon+\frac{8 \mathcal{K}}{\theta^{2}}+\mathcal{K}\right)\left|\mathfrak{G}_{m}(1 ; \rho)-1\right| \\
+ & \frac{16 \mathcal{K}}{\theta^{2}}\left|\mathfrak{G}_{m}(r ; \rho)-\rho\right|+\frac{8 \mathcal{K}}{\theta^{2}}\left|\mathfrak{G}_{m}\left(r^{2} ; \rho\right)-\rho^{2}\right| \\
\leqq & \mathcal{A}\left(\left|\mathfrak{G}_{m}(1 ; \rho)-1\right|+\left|\mathfrak{G}_{m}(r ; \rho)-\rho\right|\right. \\
& \left.\quad+\left|\mathfrak{G}_{m}\left(r^{2} ; \rho\right)-\rho^{2}\right|\right),
\end{aligned}
$$

where

$$
\mathcal{A}=\max \left(\epsilon+\frac{8 \mathcal{K}}{\theta^{2}}+\mathcal{K}, \frac{16 \mathcal{K}}{\theta^{2}}, \frac{8 \mathcal{K}}{\theta^{2}}\right) .
$$

Now, for a given $\omega>0$, there exists $\epsilon>0(\epsilon<\omega)$ such that

$$
\mathfrak{T}_{m}(\rho ; \omega)=\left\{m: m \leqq P_{k} \quad \text { and } \quad p_{m}\left|\mathfrak{G}_{m}(h ; \rho)-h(\rho)\right| \geqq \omega\right\} .
$$

Furthermore, for $v=0,1,2$, we have

$$
\mathfrak{T}_{v, m}(\rho ; \omega)=\left\{m: m \leqq P_{k} \quad \text { and } \quad p_{m}\left|\mathfrak{G}_{m}(h ; \rho)-h_{v}(\rho)\right| \geqq \frac{\omega-\epsilon}{3 \mathcal{A}}\right\},
$$

so that

$$
\mathfrak{T}_{m}(\rho ; \omega) \leqq \sum_{\nu=0}^{2} \mathfrak{T}_{v, m}(\rho ; \omega)
$$

Clearly, we obtain

$$
\frac{\left\|\mathfrak{T}_{m}(\rho ; \omega)\right\|_{\mathcal{C}[0,1]}}{P_{k}} \leqq \sum_{\nu=0}^{2} \frac{\left\|\mathfrak{T}_{v, m}(\rho ; \omega)\right\|_{\mathcal{C}[0,1]}}{P_{k}}
$$

Now, using the above assumption about the implications in (4) to (6) and by Definition 3 , the right-hand side of (15) tends to zero as $n \rightarrow \infty$. Consequently, we get

$$
\lim _{k \rightarrow \infty} \frac{\left\|\mathfrak{T}_{m}(\rho ; \omega)\right\|_{\mathcal{C}[0,1]}}{P_{k}}=0(\delta, \omega>0) .
$$

Therefore, the implication (3) holds true. This completes the proof of Theorem 4. 
Theorem 5. Let

$$
\mathfrak{G}_{j}: C[0,1] \rightarrow C[0,1]
$$

be a sequence of positive linear operators. Then, for all $h \in C[0,1]$,

$$
\text { stat }_{\text {DWR }} \lim _{j \rightarrow \infty}\left\|\mathfrak{G}_{j}(h ; \rho)-h(\rho)\right\|_{\infty}=0
$$

if and only if

$$
\begin{aligned}
& \text { stat }_{\text {DWR }} \lim _{j \rightarrow \infty}\left\|\mathfrak{G}_{j}(1 ; \rho)-1\right\|_{\infty}=0, \\
& \text { stat }_{\text {DWR }} \lim _{j \rightarrow \infty}\left\|\mathfrak{G}_{j}(\rho ; \rho)-\rho\right\|_{\infty}=0
\end{aligned}
$$

and

$$
\text { stat }_{\text {DWR }} \lim _{j \rightarrow \infty}\left\|\mathfrak{G}_{j}\left(\rho^{2} ; \rho\right)-\rho^{2}\right\|_{\infty}=0
$$

Proof. Theorem 5 can be proved in the similar lines of the proof of Theorem 4 . Therefore, we choose to skip the details involved.

In view of Theorem 5, we consider here an example that, a sequence of positive linear operators which does not work via the statistical versions of the deferred weighted Riemann integrable functions (Theorem 4). Nevertheless, it fairly works on Theorem 5. In this sense we say, Theorem 5 is a non-trivial generalization of the statistical weighted Riemann integrable functions (Theorem 4).

We now think of the operator

$$
\rho(1+\rho D) \quad\left(D=\frac{d}{d \rho}\right)
$$

that was used by Al-Salam [21], and subsequently, by Viskov and Srivastava [22].

Example 4. Consider the Bernstein polynomial $\mathfrak{B}_{n}(h ; \beta)$ on $C[0,1]$ given by

$$
\mathfrak{B}_{k}(h ; \beta)=\sum_{\varrho=0}^{k} f\left(\frac{\varrho}{k}\right)\left(\begin{array}{l}
k \\
\varrho
\end{array}\right) \beta^{\varrho}(1-b)^{k-\varrho} \quad(\beta \in[0,1] ; k=0,1, \cdots) .
$$

We now approach the positive linear operators on $C[0,1]$ is given by (20) as follows:

$$
\mathfrak{G}_{\varrho}(h ; \beta)=\left[1+h_{\varrho}\right] \beta(1+\beta D) \mathfrak{B}_{\varrho}(h ; \beta) \quad(\forall h \in C[0,1]),
$$

where $\left(h_{\rho}\right)$ is the sequence of functionds given in Example 2.

We now determine the values of each of the testing functions $1, \beta$ and $\beta^{2}$ by using (22) as follows:

$$
\begin{gathered}
\mathfrak{G}_{\varrho}(1 ; \beta)=\left[1+h_{\varrho}\right] \beta(1+\beta D) 1=\left[1+h_{\varrho}\right] \beta, \\
\mathfrak{G}_{\varrho}(t ; \beta)=\left[1+h_{\varrho}\right] \beta(1+\beta D) \beta=\left[1+h_{\varrho}\right] \beta(1+\beta)
\end{gathered}
$$

and

$$
\begin{aligned}
\mathfrak{G}_{\varrho}\left(t^{2} ; \beta\right) & =\left[1+h_{\varrho}\right] \beta(1+\beta D)\left\{\beta^{2}+\frac{\beta(1-\beta)}{\varrho}\right\} \\
& =\left[1+h_{\varrho}\right]\left\{\beta^{2}\left(2-\frac{3 \beta}{\varrho}\right)\right\} .
\end{aligned}
$$


Consequently, we have

$$
\begin{aligned}
& \text { stat }_{\text {DWR }} \lim _{\varrho \rightarrow \infty}\left\|\mathfrak{G}_{\varrho}(1 ; \beta)-1\right\|_{\infty}=0, \\
& \text { stat }_{\text {DWR }} \lim _{\varrho \rightarrow \infty}\left\|\mathfrak{G}_{\varrho}(\beta ; \beta)-\beta\right\|_{\infty}=0
\end{aligned}
$$

and

$$
\operatorname{stat}_{\mathrm{DWR}} \lim _{\varrho \rightarrow \infty}\left\|\mathfrak{G}_{\varrho}\left(\beta^{2} ; \beta\right)-\beta^{2}\right\|_{\infty}=0,
$$

that is, the sequence $\mathfrak{G}_{\varrho}(h ; \beta)$ satisfies the conditions (17) to (19). Therefore, by Theorem 5 , we have

$$
\operatorname{stat}_{\mathrm{DWR}} \lim _{\varrho \rightarrow \infty}\left\|\mathfrak{G}_{\varrho}(h ; \beta)-h\right\|_{\infty}=0 .
$$

The given sequence $\left(h_{k}\right)$ of functions mentioned as in Example 2 is statistically deferred weighted Riemann summable, but not deferred weighted statistically Riemann integrable. Therefore, our proposed operators defined by (22) satisfy Theorem 5. However, they do not satisfy for statistical versions of deferred weighted Riemann integrable functions (Theorem 4).

Now in view of our proposed mean (2), we use the notions of statistical Lebesgue integrability (DWL stat $_{\text {) }}$ and statistical Lebesgue summability (stat ${ }_{\mathrm{DWL}}$ ) for sequence of measurable functions to establish the following Korovkin-type approximation theorems.

Theorem 6. Let

$$
\mathfrak{G}_{j}: C[0,1] \rightarrow C[0,1]
$$

be a sequence of positive linear operators. Then, for all $h \in C[0,1]$,

$$
\mathrm{DWL}_{\text {stat }} \lim _{j \rightarrow \infty}\left\|\mathfrak{G}_{j}(h ; \rho)-h(\rho)\right\|_{\infty}=0
$$

if and only if

$$
\begin{aligned}
& \text { DWL }_{\text {stat }} \lim _{j \rightarrow \infty}\left\|\mathfrak{G}_{j}(1 ; \rho)-1\right\|_{\infty}=0, \\
& \text { DWL } L_{\text {stat }} \lim _{j \rightarrow \infty}\left\|\mathfrak{G}_{j}(\rho ; \rho)-\rho\right\|_{\infty}=0
\end{aligned}
$$

and

$$
\text { DWL }_{\text {stat }} \lim _{j \rightarrow \infty}\left\|\mathfrak{G}_{j}\left(\rho^{2} ; \rho\right)-\rho^{2}\right\|_{\infty}=0
$$

Proof. Theorem 6 can be proved in the similar lines of the proof of Theorem 4 . Therefore, we choose to skip the details involved.

Theorem 7. Let

$$
\mathfrak{G}_{j}: C[0,1] \rightarrow C[0,1]
$$

be a sequence of positive linear operators. Then, for all $h \in C[0,1]$,

$$
\operatorname{stat}_{\mathrm{DWL}} \lim _{j \rightarrow \infty}\left\|\mathfrak{G}_{j}(h ; \rho)-h(\rho)\right\|_{\infty}=0
$$

if and only if

$$
\begin{aligned}
& \operatorname{stat}_{\text {DWR }} \lim _{j \rightarrow \infty}\left\|\mathfrak{G}_{j}(1 ; \rho)-1\right\|_{\infty}=0, \\
& \text { stat }_{\text {DWR }} \lim _{j \rightarrow \infty}\left\|\mathfrak{G}_{j}(\rho ; \rho)-\rho\right\|_{\infty}=0
\end{aligned}
$$


and

$$
\operatorname{stat}_{\text {DWR }} \lim _{j \rightarrow \infty}\left\|\mathfrak{G}_{j}\left(\rho^{2} ; \rho\right)-\rho^{2}\right\|_{\infty}=0
$$

Proof. Theorem 7 can be proved in the similar lines of the proof of Theorem 4 . Therefore, we choose to skip the details involved.

In view of Theorem 7, we consider here an example that, a sequence of positive linear operators which does not work via the statistical versions of the deferred weighted Lebesgue integrable sequence of measurable functions (Theorem 6). Nevertheless, it fairly works on Theorem 7. In this sense, we say Theorem 7 is a non-trivial generalization of the statistical deferred weighted Lebesgue integrable sequence of measurable functions (Theorem 6).

Example 5. Consider the Bernstein polynomial $\mathfrak{B}_{n}(h ; \beta)$ on $C[0,1]$ given by (21).

We now approach the positive linear operators on $C[0,1]$ under the composition of (21) and (20) as follows:

$$
\mathfrak{H}_{\varrho}(h ; \beta)=\left[1+h_{\varrho}\right] \beta(1+\beta D) \mathfrak{B}_{\varrho}(h ; \beta) \quad(\forall h \in C[0,1]),
$$

where $\left(h_{\varrho}\right)$ is the same as mentioned in Example 3.

In similar lines of Example 4, we determine the values of each of the testing functions $1, \beta$ and $\beta^{2}$ by using (34) as follows:

$$
\begin{gathered}
\mathfrak{H}_{\varrho}(1 ; \beta)=\left[1+h_{\varrho}\right] \beta(1+\beta D) 1=\left[1+h_{\varrho}\right] \beta, \\
\mathfrak{H}_{\varrho}(t ; \beta)=\left[1+h_{\varrho}\right] \beta(1+\beta D) \beta=\left[1+h_{\varrho}\right] \beta(1+\beta)
\end{gathered}
$$

and

$$
\begin{aligned}
\mathfrak{H}_{\varrho}\left(t^{2} ; \beta\right) & =\left[1+h_{\varrho}\right] \beta(1+\beta D)\left\{\beta^{2}+\frac{\beta(1-\beta)}{\varrho}\right\} \\
& =\left[1+h_{\varrho}\right]\left\{\beta^{2}\left(2-\frac{3 \beta}{\varrho}\right)\right\} .
\end{aligned}
$$

Consequently, we have

$$
\begin{aligned}
& \operatorname{stat}_{\text {DWL }} \lim _{\varrho \rightarrow \infty}\left\|\mathfrak{H}_{\varrho}(1 ; \beta)-1\right\|_{\infty}=0, \\
& \operatorname{stat}_{\text {DWL }} \lim _{\varrho \rightarrow \infty}\left\|\mathfrak{G}_{\varrho}(\beta ; \beta)-\beta\right\|_{\infty}=0
\end{aligned}
$$

and

$$
\operatorname{stat}_{\text {DWL }} \lim _{\varrho \rightarrow \infty}\left\|\mathfrak{H}_{\varrho}\left(\beta^{2} ; \beta\right)-\beta^{2}\right\|_{\infty}=0,
$$

that is, the sequence $\mathfrak{H}_{Q}(h ; \beta)$ satisfies the conditions (35) to (37). Therefore, by Theorem 7 , we have

$$
\operatorname{stat}_{\text {DWR }} \lim _{\varrho \rightarrow \infty}\left\|\mathfrak{H}_{\varrho}(h ; \beta)-h\right\|_{\infty}=0 .
$$

The given sequence of the functions $\left(h_{k}\right)$ mentioned in Example 3 is statistically deferred weighted Lebesgue summable, but not deferred weighted statistically Lebesgue integrable. Therefore, our proposed operators defined by (34) satisfy Theorem 7 . However, they do not satisfy for statistical versions of deferred weighted Lebesgue integrable sequence of functions (Theorem 6). 


\section{Remarkable Conclusion}

In this concluding section of our investigation, we further observe the potentiality of our Theorems 5 and 7 over Theorems 4 and 6 respectively, and in general, over the classical versions of the Korovkin-type approximation theorems.

Remark 1. Let us consider the sequence of functions $\left(h_{\varrho}\right)_{\varrho \in \mathbb{N}}$ as in Example 2 and also that $\left(h_{\varrho}\right)$ is statistically deferred weighted Riemann summable, so that

$$
\text { stat }_{\mathrm{DWR}} \lim _{\varrho \rightarrow \infty} \delta\left(h_{\varrho} ; \mathcal{P}\right)=\frac{1}{2} \text { on }[0,1] .
$$

Then we have

$$
\text { stat }_{\mathrm{DWR}} \lim _{k \rightarrow \infty}\left\|\mathfrak{G}_{k}\left(h_{v} ; \rho\right)-f_{v}(\rho)\right\|_{\infty}=0 \quad(v=0,1,2) .
$$

Thus, by Theorem 5, we immediately get

$$
\text { stat }_{\text {DWR }} \lim _{j \rightarrow \infty}\left\|\mathfrak{G}_{k}(h ; \rho)-h(\rho)\right\|_{\infty}=0,
$$

where

$$
h_{0}(\rho)=1, \quad h_{1}(\rho)=\rho \text { and } h_{2}(\rho)=\rho^{2} .
$$

Here the functions $\left(h_{k}\right)$ is statistically deferred weighted Riemann summable, but neither Riemann integrable nor deferred weighted statistically Riemann integrable. Thus, this application evidently demonstrates that our Korovkin-type approximation Theorem 5 properly works under the operators defined in the Equation (22), but the statistical versions as well as classical of deferred weighted Riemann integrable functions do not behave properly for the same operators. In view of this observation, we certainly say that our Theorem 5 is a non-trivial generalization of Theorem 4 as well as the classical Korovkin-type approximation theorem [23].

Remark 2. Let us consider the functions $\left(h_{\varrho}\right)_{\varrho \in \mathbb{N}}$ in Example 3 and also that $\left(h_{\varrho}\right)$ is statistically deferred weighted Lebesgue summable, so that

$$
\operatorname{stat}_{\mathrm{DWL}} \lim _{\varrho \rightarrow \infty} b_{\varrho} \lambda\left(B_{\varrho}\right)=\frac{1}{2} \text { on }[0,1] .
$$

Then, we have

$$
\text { stat }_{\mathrm{DWL}} \lim _{k \rightarrow \infty}\left\|\mathfrak{G}_{k}\left(h_{v} ; \rho\right)-h_{v}(\rho)\right\|_{\infty}=0 \quad(v=0,1,2) .
$$

Thus by Theorem 7, we immediately get

$$
\text { stat }_{\mathrm{DWL}} \lim _{k \rightarrow \infty}\left\|\mathfrak{G}_{k}(h ; \rho)-h(\rho)\right\|_{\infty}=0,
$$

where

$$
h_{0}(\rho)=1, \quad h_{1}(\rho)=\rho \text { and } h_{2}(\rho)=\rho^{2} .
$$

As we known $\left(h_{k}\right)$ is statistically deferred weighted Lebesgue summable, but neither classically Lebesgue integrable nor statistically Lebesgue integrable via deferred weighted mean. Therefore, our Korovkin-type approximation Theorem 7 properly works under the operators defined in the Equation (34), but the statistical versions as well as classical version of deferred weighted Lebesgue integrable functions do not behave properly for the same operators. In view of this observation, we certainly say that our Theorem 7 is a non-trivial generalization of Theorem 6 as well as the classical Korovkin-type approximation theorem [23]. 
Remark 3. In view of all the Korovkin-type approximation theorems established in Section 4, we certainly set-up a containments zone, Theorem $4 \subseteq$ Theorem $5 \subseteq$ Theorem $6 \subseteq$ Theorem 7 . That is, Theorem 7 generalizes rest other theorems.

Remark 4. Influenced by a recently-published survey-cum-expository review article by Srivastava [24], we draw the awareness of curious reader's toward the prospect of exploring the qgeneralizations of the results which are demonstrated in this paper. Moreover, the $(p, q)$-extension will be a fairly trivial and insignificant change based on the proposed extension as the the additional parameter $p$ is redundant (see, for details, Srivastava [24], p. 340). Furthermore, in view of a recent result of Srivastava [25], the attention of the interested reader's is also drawn for further researches towards $(k, s)$-extension of the Riemann-Liouville fractional integral.

Author Contributions: Formal analysis, H.M.S. and S.K.P.; Investigation, B.B.J.; Methodology, S.K.P.; Supervision, H.M.S. and S.K.P.; Writing-original draft, B.B.J.; Writing-review \& editing, S.K.P. All authors have read and agreed to the published version of the manuscript.

Funding: This research received no external funding.

Conflicts of Interest: The authors declare no conflict of interest.

\section{References}

1. Fast, H. Sur la convergence statistique. Colloq. Math. 1951, 2, 241-244. [CrossRef]

2. Steinhaus, H. Sur la convergence ordinaire et la convergence asymptotique. Colloq. Math. 1951, 2, 73-74.

3. Altomare, F. Korovkin-type theorems and approximation by positive linear operators. Surv. Approx. Theory 2010, 5, 92-164.

4. Akdag, S. Weighted equi-statistical convergence of the Korovkin-type approximation theorems. Results Math. 2017, 72, $1073-1085$. [CrossRef]

5. Guessab, A.; Schmeisser, G. Two Korovkin-type theorems in multivariate approximation. Banach J. Math. Anal. 2008, 2, 121-128. [CrossRef]

6. Guessab, A.; Schmeisser, G. Convexity results and sharp error estimates in approximate multivariate integration. Math. Comp. 2004, 73, 1365-1384. [CrossRef]

7. Móricz, F. Tauberian conditions under which statistical convergence follows from statistical summability (C,1). J. Math. Anal. Appl. 2002, 275, 277-287. [CrossRef]

8. Mohiuddine, S.A.; Alotaibi, A.; Mursaleen, M. Statistical summability $(C, 1)$ and a Korovkin-type approximation theorem. J. Inequal. Appl. 2012, 2012, 1-8. [CrossRef]

9. Karakaya, V.; Chishti, T.A. Weighted statistical convergence. Iran. J. Sci. Technol. Trans. A Sci. 2009, 33, $219-223$.

10. Mursaleen, M.; Karakaya, V.; Ertürk, M.; Gürsoy, F. Weighted statistical convergence and its application to Korovkin-type approximation theorem. Appl. Math. Comput. 2012, 218, 9132-9137. [CrossRef]

11. Srivastava, H.M.; Jena, B.B.; Paikray, S.K.; Misra, U.K. A certain class of weighted statistical convergence and associated Korovkin type approximation theorems for trigonometric functions. Math. Methods Appl. Sci. 2018, 41, 671-683. [CrossRef]

12. Srivastava, H.M.; Jena, B.B.; Paikray, S.K.; Misra, U.K. Generalized equi-statistical convergence of the deferred Nörlund summability and its applications to associated approximation theorems. Rev. Real Acad. Cienc. Exactas Fís. Natur. Ser. A Mat. (RACSAM) 2018, 112, 1487-1501. [CrossRef]

13. Dutta, H.; Paikray, S.K.; Jena, B.B. On statistical deferred Cesàro summability. In Current Trends in Mathematical Analysis and Its Interdisciplinary Applications; Dutta, H., Ljubiša Kočinac, D.R., Srivastava, H.M., Eds.; Springer Nature, Switzerland AG: Cham, Switzerland, 2019; pp. 885-909.

14. Balcerzak, M.; Dems, K.; Komisarski, A. Statistical convergence and ideal convergence for sequences of functions. J. Math. Anal. Appl. 2007, 328, 715-729. [CrossRef]

15. Braha, N.L. Some weighted equi-statistical convergence and Korovkin type-theorem. Results Math. 2016, 70, 433-446. [CrossRef]

16. Jena, B.B.; Paikray, S.K.; Dutta, H. Statistically Riemann integrable and summable sequence of functions via deferred Cesàro mean. Bull. Iran. Math. Soc. 2021, 1-17. [CrossRef]

17. Karakuş, S.; Demirci, K.; Duman, O. Equi-statistical convergence of positive linear operators. J. Math. Anal. Appl. 2008, 339, 1065-1072. [CrossRef]

18. Mohiuddine, S.A. An application of almost convergence in approximation theorems. Appl. Math. Lett. 2011, 24, 1856-1860. [CrossRef]

19. Srivastava, H.M.; Jena, B.B.; Paikray, S.K. A certain class of statistical probability convergence and its applications to approximation theorems. Appl. Anal. Discret. Math. 2020, 14, 579-598. [CrossRef]

20. Srivastava, H.M.; Jena, B.B.; Paikray, S.K. Statistical deferred Nörlund summability and Korovkin-type approximation theorem. Mathematics 2020, 8, 636. [CrossRef]

21. Al-Salam, W.A. Operational representations for the Laguerre and other polynomials. Duke Math. J. 1964, 31, 127-142. [CrossRef] 
22. Viskov, O.V.; Srivastava, H.M. New approaches to certain identities involving differential operators. J. Math. Anal. Appl. 1994, 186, 1-10. [CrossRef]

23. Korovkin, P.P. Convergence of linear positive operators in the spaces of continuous functions (in Russian). Doklady Akad. Nauk. SSSR (New Ser.) 1953, 90, 961-964.

24. Srivastava, H.M. Operators of basic (or $q$-) calculus and fractional $q$-calculus and their applications in geometric function theory of complex analysis. Iran. J. Sci. Technol. Trans. A Sci. 2020, 44, 327-344. [CrossRef]

25. Srivastava, H.M. Some parametric and argument variations of the operators of fractional calculus and related special functions and integral transformatioons. J. Nonlinear Convex Anal. 2021, 22, 1501-1520. 